\title{
Joint effects of group sex-ratio and Wolbachia infection on female reproductive success in the terrestrial isopod Armadillidium vulgare
}

Margot Fortin ${ }^{1}$, Joël Meunier ${ }^{2}$, Tiffany Laverré ${ }^{1}$, Catherine Souty-Grosset ${ }^{1}$ and Freddie-Jeanne Richard ${ }^{1 *}$ (D)

\begin{abstract}
Background: In species that reproduce with sexual reproduction, males and females often have opposite strategies to maximize their own fitness. For instance, males are typically expected to maximize their number of mating events, whereas an excessive number of mating events can be costly for females. Although the risk of sexual harassment by males and resulting costs for females are expected to increase with the proportion of males, it remains unknown whether and how parasitic distorters of a host population's sex-ratio can shape this effect on the fitness of females. Here, we addressed this question using Armadillidium vulgare and its parasite Wolbachia that alters the sexratio of a population. We set up Wolbachia-free and Wolbachia-infected females in experimental groups exhibiting 100, $80,50 \%$ or $20 \%$ females for 1 year, during which we measured changes in survival, fertility and fecundity.
\end{abstract}

Results: Wolbachia infection shaped the effects of both population sex-ratio and reproductive season on female fecundity. Compared to Wolbachia-free females, Wolbachia-infected females were less likely to be gravid in populations exhibiting an excess of females and did not exhibit the otherwise negative effect of seasons on this likelihood. Group sex-ratio and Wolbachia infection have independent effects on other measured traits. Male-biased populations had females both exhibiting the lowest survival rate after 6 months and producing the smallest number of offspring, independent of Wolbachia infection. Conversely, Wolbachia-infected females had the lowest likelihood of producing at least one offspring, independent of group sex-ratio. Wolbachia infection had no effect on female survival rate.

Conclusions: We demonstrated that male-biased sex-ratio and the presence of Wolbachia are costly for females due to sexual harassment by males and bacterial infection, respectively. Interestingly, Wolbachia infection triggers another negative effect. This effect does not come from an excess of males and its associated sexual harassment of females but instead from a lack of males and the associated risk for females of remaining unmated. Overall, these findings highlight the importance of social pressures and infection on female fitness and provide insights into our general understanding of the joint and opposite effects of these two parameters in the evolution of reproductive strategies.

Keywords: Symbiosis, Mate choice, Multiple mating, Breeding successes

\footnotetext{
* Correspondence: freddie.jeanne.richard@univ-poitiers.fr

${ }^{1}$ Laboratoire Ecologie et Biologie des Interactions, Equipe "Ecologie,

Evolution, Symbiose", UMR CNRS 7267, Université de Poitiers, Bat B8-B35, 6

rue Michel Brunet, TSA 51106, F-86073 Poitiers, Cedex 9, France

Full list of author information is available at the end of the article
}

(c) The Author(s). 2019 Open Access This article is distributed under the terms of the Creative Commons Attribution 4.0 International License (http://creativecommons.org/licenses/by/4.0/), which permits unrestricted use, distribution, and reproduction in any medium, provided you give appropriate credit to the original author(s) and the source, provide a link to the Creative Commons license, and indicate if changes were made. The Creative Commons Public Domain Dedication waiver (http://creativecommons.org/publicdomain/zero/1.0/) applies to the data made available in this article, unless otherwise stated. 


\section{Background}

Because males and females typically differ in their investment in reproduction, sexual conflict is predicted to emerge in species with sexual reproduction [1]. In this context, males often improve their fitness by maximizing the number of their sexual partners, whereas females do so by being selective toward the quality of their mate $[2,3]$. This sex-specific difference in selective pressure has been suggested to lead to sexual harassment by males, a process during which males constrain females for reproduction and which increases the mating costs for females [3-5]. On the one hand, harassment by males may impact female fitness indirectly, for example by forcing females to spend more time and energy avoiding mating instead of investing in other activities such as foraging [6-8]. On the other hand, harassment by males may have a direct impact on female fitness; for instance, in the bean weevil Callosobruchus maculatus, the genitalia of males have been shown to contain many sclerotized spines, damaging genitalia of females during copulation and therefore increasing the mortality of females after multiple mating events [4]. Direct costs have also been reported in the sea lion Phocarctos hookeri, where sexual harassment by males increases both female mortality and the associated costs of pup separation [8].

The expression of sexual harassment by males and its resulting costs for females may strongly depend on the proportion of males in a population, as well as on the presence of parasitic distorters, such as the symbiotic bacteria Wolbachia, on the host population's sex-ratio. For example, the importance of male-biased sex-ratio on the sexual harassment of females has been shown in the water strider Aquarius remiges. In this species, an artificially male-biased sex-ratio increased the quantity of sexual harassment by males, which in turn modified the mating behavior of females; they invested more time in escape behaviors from males by reducing their foraging time [7]. Conversely, numerous studies have demonstrated the broad impacts of Wolbachia - a group of intracellular alpha proteobacteria - on multiple aspects of the biology of their hosts, including their reproductive strategies and outcomes [9]. Wolbachia are some of the most prevalent gram-negative bacteria in arthropods [10]. They are vertically transmitted and highly manipulative symbionts [11-14], which trigger multiple effects on the reproduction of their host, such as the emergence of cytoplasmic incompatibilities, induction of parthenogenesis, killing of males and feminization of genetic males [15]. In the terrestrial isopod Armadillidium vulgare (Crustacea, Isopoda), Wolbachia infected individuals suffer from important fitness costs, such as reduced density and survival of hemocytes [16], lower learning and memory performances, lower copulation investment [17], and reduced preference of infected females by males [18].
In this species, Wolbachia is also known to induce the feminization of genetic males by transforming them into phenotypic and functional females $[19,20]$. Variation in the prevalence of Wolbachia infection across natural populations could thus shape their sex-ratio [15], and Wolbachia could therefore have important effects on the expression of sexual harassment by males and on its resulting costs for females.

In this study, we manipulated male-biased sex-ratios and Wolbachia infection in four types of experimental groups to test their independent and simultaneous effects on the survival and reproduction of $A$. vulgare females. In this species, females can produce several clutches from only one copulation [21], even if multiple mating frequently occurs [22-24]. Females also have several periods of receptivity throughout their life, as well as exhibit refractory periods just after a first mating and after a first breeding season [24, 25]. At the same time, males can mate with numerous females in a few days [26].

We maintained Wolbachia-free or Wolbachia-infected females in experimental groups including either 100, 80, $50 \%$ or $20 \%$ females, and then recorded their survival rate, fertility (gravidity) and fecundity (offspring number) over the following 12 months. If sexual harassment by males is overall costly for females while increasing their mating probability (by providing more mating partners and thus increasing overall sperm availability), we predicted that increased male density should overall negatively affect female survival and increase both their fertility and fecundity. Because Wolbachia negatively affects the immune system and survival of its hosts [16], we expected the additional energetic costs of reproductive effort (e.g., male injury, physiological changes due to offspring development) to be reduced in Wolbachia-free compared to Wolbachia-infected females for survival, fertility and fecundity.

\section{Results \\ Effects of group sex-ratio and Wolbachia infection on the survival rate and reproductive success of females Female survival rates}

Over the first 6 months of the experiment, the survival rate of females depended on the group sex-ratio (Fig. 1a; Likelihood Ratio (LR) $X^{2}{ }_{3}=40.74, P<0.0001$ ) but was independent of Wolbachia infection (Fig. 1b; LR $X^{2}{ }_{1}=1.76$, $P=0.1849)$ and the interaction between group sex-ratio and Wolbachia infection (LR $\left.X_{3}^{2}=7.04, P=0.071\right)$. In particular, Females 20\% died overall faster than Females $50 \%$ (model contrasts; LR $X^{2}{ }_{1}=14.58, P=0.0001$ ), Females $80 \%$ (LR $\left.X_{1}{ }_{1}=26.54, P<0.0001\right)$ and Females ${ }_{100 \%}\left(\operatorname{LR} X^{2}{ }_{1}=\right.$ 22.26, $P<0.0001$ ). The survival rates of Females $50 \%$, Females $80 \%$ and Females $100 \%$ were similar during this time period (Females $50 \%$ vs Females $80 \%$ : LR $\chi^{2}{ }_{1}=2.70$, 

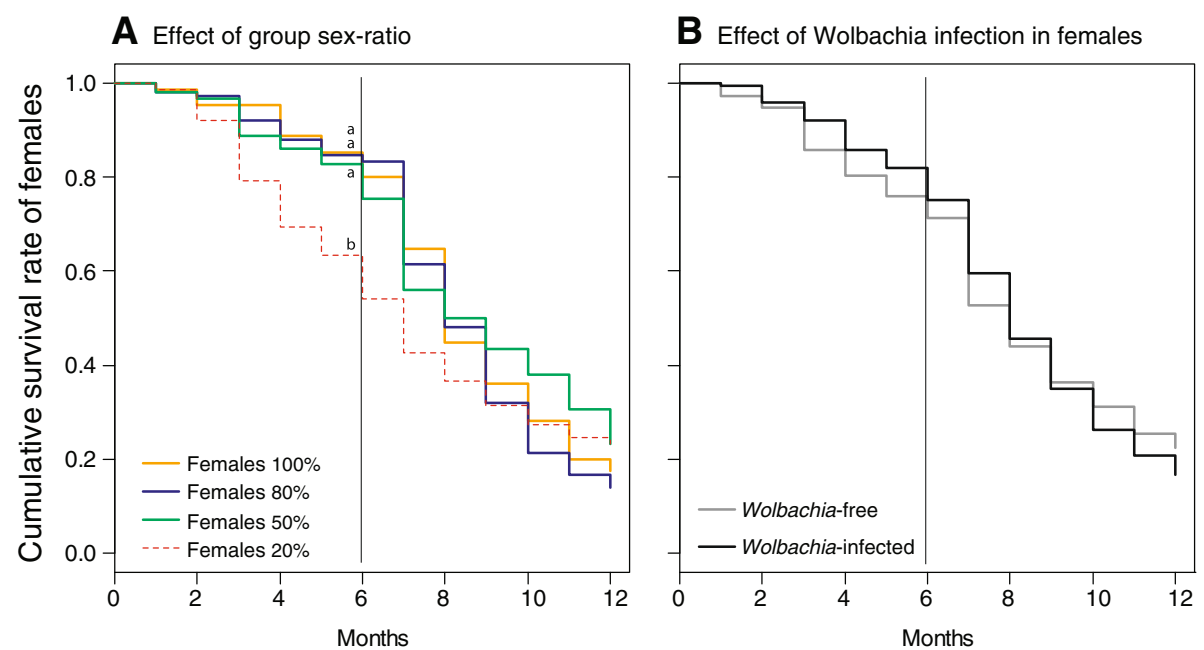

Fig. 1 Cumulative survival rates of females over 12 months as a function of (a) group sex-ratio and (b) Wolbachia infection in females. Different letters represent $P<0.001$ six months after the beginning of the experiment

$P=0.100$; Females $50 \%$ vs Females ${ }_{100 \%}$ : $L R X^{2}{ }_{1}=1.08$, $P=0.300$; Females $80 \%$ vs Females ${ }_{100 \%}$ : LR $\chi^{2}{ }_{1}=0.39$, $P=0.530)$. Interestingly, these effects vanished when the overall 12 months of the experiment were considered. In this case, the survival of females became independent of group sex-ratio (LR $X_{3}^{2}=4.05, P=0.2558$ ), Wolbachia infection ( $\left.\operatorname{LR} X^{2}{ }_{1}=0.31, P=0.5755\right)$ and the interaction between group sex-ratio and Wolbachia infection (LR $X_{3}^{2}$ $=2.67, P=0.4447)$.

\section{Proportion of gravid females}

The proportion of gravid females was shaped by a triple interaction between Wolbachia infection, group sex-ratio and month (LR $x_{3}^{2}=8.96, p=0.0297$; see full model results in Additional file 1: Table S1). This interaction revealed that in Wolbachia-free females, the proportion of gravid females overall decreased over time (Fig. 2a; LR $\chi^{2}{ }_{1}=8.59, P=0.0034$; model estimate $\pm \mathrm{SE}=$ $-0.042 \pm 0.013)$ but was independent of group sex-ratio (LR $\left.X_{3}^{2}=0.15, P=0.9849\right)$ and the interaction between group sex-ratio and month $\left(\operatorname{LR} X^{2}{ }_{3}=4.39, P=0.2227\right)$. In contrast, in Wolbachia-infected females, the proportion of gravid females did not vary over time (Fig. $2 \mathrm{~b}$; $\mathrm{LR} \chi_{{ }_{1}{ }_{1}=}$ 3.53, $P=0.0602$ ) but reflected group sex-ratio overall (LR $\left.X_{3}^{2}=13.75, P=0.0033\right)$. In particular, the proportion of gravid females was lower in Females $80 \%$ compared with Females 20\% (Tukey contrasts, $P=0.0299$ ) and Females $50 \%$ (Tukey contrasts, $P=0.0013$ ), whereas all the other pairwise comparisons were nonsignificant (Fig. 2a; all $P>0.1450)$. The proportion of gravid Wolbachia-infected females was independent of the interaction between group sex-ratio and month $\left(\operatorname{LR} X^{2}{ }_{3}=7.65, P=0.0539\right)$.

\section{Production of descendants}

The proportion of females (alive) producing at least one pullus during our monthly observations was overall higher in Wolbachia-free compared to Wolbachia-infected females (Fig. 3a; LR $X^{2}{ }_{1}=18.91, P<0.0001$ ), as well as overall increased over time $\left(\operatorname{LR} \chi^{2}{ }_{1}=44.22, P<0.0001\right.$; model estimate $\pm \mathrm{SE}=1.00 \pm 0.15)$. However, this proportion was independent of group sex-ratio ( $\mathrm{LR} X^{2}{ }_{2}=1.94, P=0.380$ ) and of any interaction among the three tested factors (all $P>0.0709)$.

Overall, the mean number of descendants produced by each of these reproductive females (i.e., producing at least one pullus) decreased over the season (Fig. 3b; LR $X_{1}^{2}=82.80, P<0.0001$; model estimate $\pm \mathrm{SE}=-$ $0.88 \pm 0.09)$ and reflected group sex-ratio (LR $X_{2}^{2}=$ 17.76, $P=0.0001)$. In particular, this mean number was higher in Females $80 \%$ compared to Females $20 \%$ (Tukey contrasts, $P=0.0001$ ), whereas there was no difference between Females $20 \%$ and Females ${ }_{50 \%}(P=0.1049)$ and between Females $50 \%$ and Females $80 \%(P=0.0914)$. In contrast, the mean number of descendants produced by these reproductive females was independent of Wolbachia infection (Fig. 3b; LR $X^{2}{ }_{1}=0.18, P=0.6711$ ). It is noteworthy that the triple interaction between Wolbachia, month and group sex ratio was not tested due to the lack of descendants produced by Wolbachia-infected females for the first 3 months.

\section{Discussion}

To our knowledge, our experimental work is the first to explore the long term and (possibly) interactive effects of group sex-ratio and Wolbachia-infections on the 

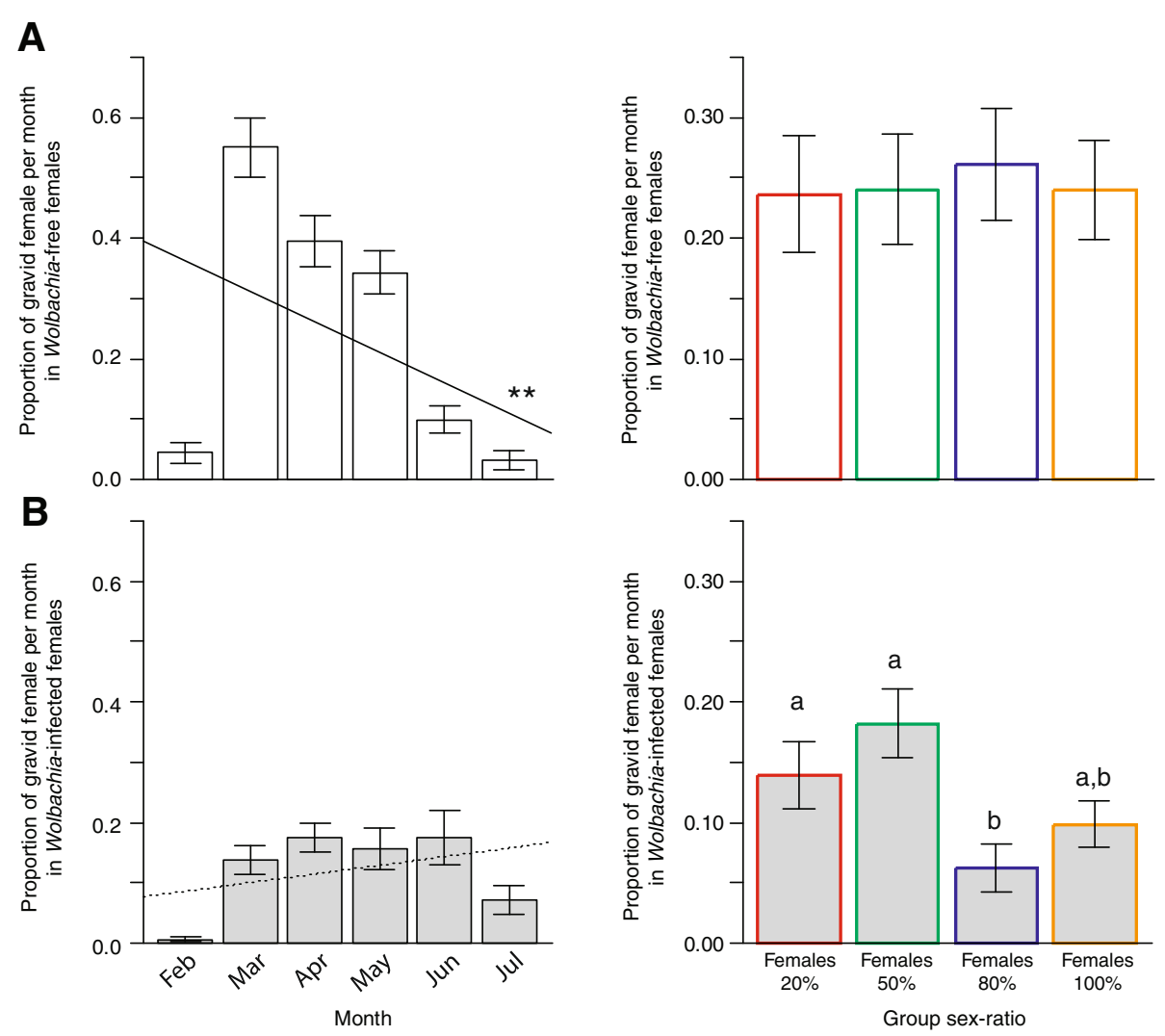

Fig. 2 Ratio of gravid females as a function of month (left) and group sex-ratio (right) in (a) Wolbachia-free and (b) Wolbachia-infected females. Mean \pm SE are presented. Different letters correspond to $P<0.05$; ${ }^{* *} P<0.0001$

survival, fertility and fecundity of females. First, our results reveal that Wolbachia infection shapes the effects of both population sex-ratio and reproductive season on female fecundity. Compared to Wolbachia-free females, Wolbachia-infected females exhibited a lower likelihood of being gravid when surrounded by a lack of males and did not exhibit the otherwise negative effect of seasons on this likelihood. Independent of Wolbachia infection, we then found that an increase in male density was overall costly for female fitness, as it decreased both their lifespan and their number of offspring. Wolbachia-in fected females, on the other hand, suffered from the presence of a high proportion of males in the group, as it reduced the number of females that produced descendants. Wolbachia infection also reduced the likelihood of females producing at least 1 offspring, whereas it did not affect the number of pulli produced (when females had at least 1 descendant) and female lifespan. Finally, our results demonstrate that the proportion of females producing at least one descendant and the mean number of descendants for these females overall increased and decreased during the reproductive season, respectively.

We showed that infection by Wolbachia altered both the dynamics of female reproduction over 6 months and the effect of male sex-ratio on their fecundity. In Wolbachia-free females, the proportion of gravid females decreased over the reproductive season and was overall independent of male sex-ratio, whereas in Wolbachia-in fected females, this proportion was constant during the reproductive season and overall lower in groups with a lack of males. This absence of seasonal-effect on the fecundity of Wolbachia-infected females likely reflects the major reduction in female fecundity observed at the beginning of the reproductive season, which remained constant and low over time. The second effect suggests that Wolbachia-infection made females more sensitive to the risk of remaining virgin in groups where they dramatically outnumbered males. This finding is supported by previous studies showing that sperm depletion occurs in A. vulgare males but only affects the fertility of Wolbachia-infected females [27], revealing that Wolbachia-in fected females are less attractive than Wolbachia-free females and such pattern is correlated with different cuticular odor profiles [18] and demonstrating that males perform fewer interactions, fewer copulations and less sperm transfer to Wolbachia-infected compared to Wolbachia-free females [17]. Therefore, Wolbachia infection triggered the negative effects of group sex-ratio 

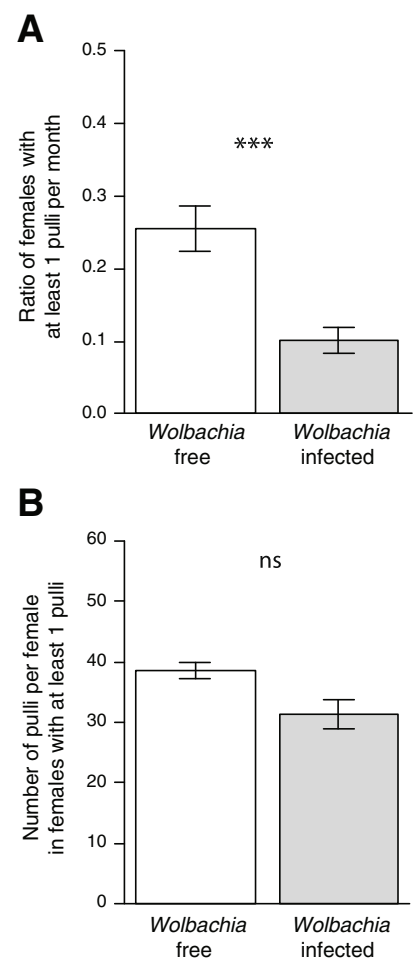
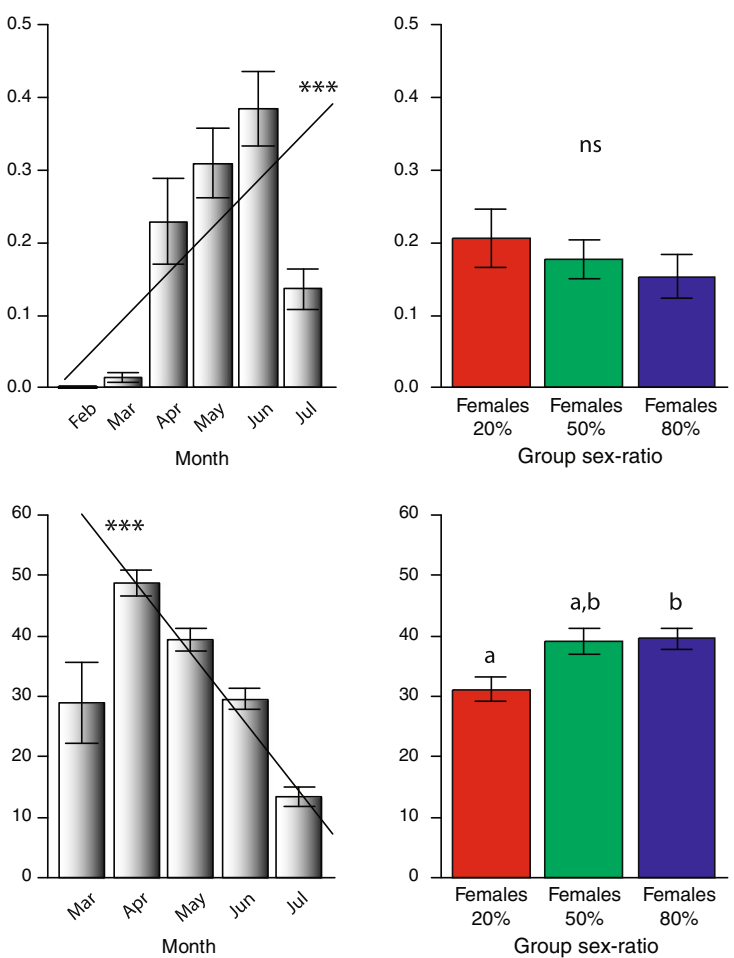

Fig. 3 Proportion of females producing at least one descendant (a) and mean number of descendants in these females (b) as a function of Wolbachia infection (left), month of observation (middle) and group sex-ratio (right). Different letters correspond to $P<0.05$; ${ }^{* * *} P<0.0001$

on female fecundity, but somewhat surprisingly, these effects did not accentuate the costs of sexual harassment for females due to male-biased sex-ratios but instead increased the risk of remaining virgin in groups exhibiting a female-biased sex-ratio.

Independent of Wolbachia-infection, our results reveal that females living with an excess of males exhibited a shorter lifespan between March and August (i.e., the onset of reproduction), as well as had lower overall reproductive success compared to the other females. Comparable effects of male-biased sex-ratios on female reproductive success have been reported in guppies and fruit flies, where females living in groups with a male-biased sex-ratio produced fewer offspring and suffered more from sexual harassment by males [28, 29]. Similarly, in guppies, the observed decrease in direct fitness was linked to sexual harassment of females and reflected their resulting reduced allocation of time to foraging behavior $[30,31]$. Our results suggest that sexual harassment by males occurred in A. vulgare and shed light on the associated fitness costs for females as suggested in a previous study [25]. Interestingly, a few observations conducted during the experiment showed that two males tried to copulate simultaneously with one female in groups with high male density, whereas this was not observed in groups with low male density.

Notwithstanding the effects of group sex-ratio reported above, our results reveal that Wolbachia-infection reduced the likelihood of females producing at least one offspring and had no effect on their total number of descendants (when produced) and on female longevity. It is generally known that the effects of Wolbachia on host fitness greatly varies between host species, Wolbachia strains and measured fitness traits. For instance, Wolbachia decreased fertility but increased the life span of individuals if they were maintained on sugar meals in Anopheles stephensi, which is not a natural host of Wolbachia [32]. Conversely, in Drosophila melanogaster, some Wolbachia strains enhanced the costs of reproduction of females living with males [33]. Somewhat surprisingly, however, we showed that Wolbachia did not affect the longevity of its host. This result is in contrast with findings from a previous study, in which the authors monitored the survival of 100 Wolbachia-free females and 100 females infected with Wolbachia, and documented a lower survival for Wolbachia-infected females [16]. However, females in this previous study were isolated with one male for reproduction. Therefore, one likely explanation for the apparent differences between these two results is the 
effect of social isolation (A. vulgare is a gregarious species), which could have hindered their investment in immunity [34] and thus strengthened the negative effects of Wolbachia on female fitness. Future studies will investigate this hypothesis.

\section{Conclusions}

To conclude, our study is the first to investigate the influence of group sex-ratio and a feminizing sex-ratio distorter on female survival and reproduction. Males, especially in a male biased sex-ratio group, appear to impact female survival and fertility, and a recent study showing that fewer females are found in natural populations at the end of the breeding season could indicate that these costs also exist under natural environments [35]. Wolbachia infection also has a strong negative impact on its host's reproductive success. Overall, our findings highlight the importance of social pressures and infection on female fitness and provide insights into our general understanding of the joint and/or opposite effects of these two parameters on the evolution of reproductive strategies.

\section{Methods}

\section{Animal rearing and experimental design}

All A. vulgare individuals (Isopoda, Oniscidea, Latreille, 1804) used in this experiment originated from a Wolbachia-free population sampled in Denmark and then were maintained under a standard laboratory condition preventing sib-mating. The Wolbachia-infected females were descendants of mothers from this population, which were initially injected with the Wolbachia $w \mathrm{VulC}$ strain [36] and then naturally transmitted their bacteria to the next generations (hereafter, the Wolbachia-in fected population). The descendants of these females are therefore feminized males and/or females [37]. In addition, all the males and Wolbachia-free females used in our experiments were derived from the Wolbachiafree population. All gravid females were isolated, their offspring then sexed, and the resulting males and females separated into different boxes before sexual maturity. All our experimental groups (see below) were set up with virgin adults that were 5-6 months old. All animals were reared in boxes $(26 \times 13 \mathrm{~cm})$ on a substrate of moistened soil, provided with ad libitum food (dead leaves and slices of fresh carrots) and maintained at $20^{\circ}$ $\mathrm{C}$ under the natural photoperiod of Poitiers (France).

\section{Effects of group sex-ratio and Wolbachia infection on the} survival rate and reproductive success of females

To test whether group sex-ratio and/or Wolbachia infection influence the survival and reproduction of females, we set up four types of groups with different sex-ratios: $100 \%$ female (15 females and 0 males), $80 \%$ female (15 females and 4 males), $50 \%$ female (15 females and 15 males) and $20 \%$ female (15 females and 60 males), hereafter called females ${ }_{100 \%}$, females ${ }_{80 \%}$, females ${ }_{50 \%}$, and females $20 \%$, respectively. For each sex-ratio, we set up five groups including Wolbachia-free females and five groups including Wolbachia-infected females. It is important to note that $A$. vulgare females can produce unfertilized eggs if they are not in contact with any males (Richard FJ, personal communication), which was therefore expected to occur in the females $100 \%$ treatment. To avoid covariation between animal density and experimental groups, the box dimensions were proportional to the number of animals inside, i.e., $17.5 \times 11.5,17.5 \times 11.5$, $35 \times 23$ and $70 \times 46 \mathrm{~cm}$, respectively. All experimental groups were then maintained under standard laboratory conditions (see above) for the following 12 months. To maintain stable sex-ratios over the experimental period, dead males were replaced by living males, whereas living males were removed from the groups where females died during these 12 months.

Female mortality was recorded on a monthly basis over the 12 months of the experiment, whereas changes in the number of gravid females per group and the total number of juveniles (called pulli) produced per female were measured over the first 6 months of the experiment, which corresponds to the reproductive period of this species. To follow the number of pulli produced per female over the reproductive season, one subset of gravid females was isolated each month in a small individual box until the birth of their pulli (i.e., just a few days). These pulli were counted, and their mothers were placed back into their experimental group. Each of these gravid females was marked on its cuticle with a Posca marker before its isolation to avoid their reuse in subsequent measurements of their groups.

\section{Data analyses}

All statistical analyses were conducted using $\mathrm{R}$ v3.4.3 (https://cran.r-project.org/) with the survival, lme4, emmeans and car packages. Initially, the survival rate of females was analyzed at 6 months (i.e., over the reproductive period) and at 12 months (i.e., over the entire experiment), that is, females still alive after six or 12 months, respectively, after the beginning of the experiment using two Cox proportional hazard regression models allowing for censored data. In these models, group sex-ratio (females ${ }_{100 \%}$, females ${ }_{80 \%}$, females $50 \%$, or females $20 \%$ ) and Wolbachia infection (Wolbachia-free or Wolbachia-infected) were the explanatory variables. To control for the nonindependence of females maintained in the same rearing boxes, we also entered the box-ID into each statistical model using the frailty argument. 
We analyzed the proportion of gravid females (females' fecundity) using a Generalized Linear Mixed Model (GLMER) with binomial error distribution and correction for overdispersion. In this model, the proportion of gravid females was entered as a response variable (using the cbind function in R), whereas group sex-ratio, Wolbachia infection and the month of observation (as a continuous value) were used as explanatory variables. The use of month as a continuous value allowed us testing whether Wolbachia-infection alters the negative effect of time on the proportion of gravid female per months. Because several females were maintained in the same rearing boxes, we also used the box-ID as a random effect.

Many females did not produce any pulli during the experiment, so female fertility was investigated in two ways. In the first way, we tested whether the proportion of females with at least 1 pullus per group reflected group sex-ratio, Wolbachia infection and the month of observation. To this end, we used a GLMER with binomial error distribution and correction for data overdispersion and entered the box-ID as a random effect. In the second step, we tested whether the number of pulli produced by these females (i.e., females with at least 1 pullus) reflected group sex-ratio, Wolbachia infection and the month of observation. Here, we used a general Linear Mixed Model (LMER) and entered the box-ID as a random effect. It is important to note that these two analyses did not include Females $100 \%$, as all these females were virgin and were consequently unable to produce fertilized eggs.

All statistical models initially included all possible interactions between the tested variables and were then simplified via the stepwise deletion of nonsignificant interactions (all $P<0.05$ ). Pairwise comparisons between group sex-ratio were tested using model contrasts and $P$-values were corrected for multiple testing using Tukey corrections.

\section{Additional files}

Additional file 1: Table S1. Full model. The proportion of gravid females was shaped by a triple interaction between Wolbachia infection, group sex-ratio and month. The full model results of the measured parameters are presented. (DOCX $12 \mathrm{~kb}$ )

Additional file 2: S2 Raw data. Data availability The excel file provides all the raw data collected in the current study. (XLSX $48 \mathrm{~kb}$ )

\section{Abbreviations}

GLMER: Generalized Linear Mixed Model; LMER: General Linear Mixed Model; LR: Likelihood Ratio

\section{Funding}

This project was also supported by the European Commission through the programme Erasmus Mundus Master Course - international Master in Applied Ecology (EMMC - IMAE) (FPA 2023-0224/ 532524-1-FR-2012-1-ERA MUNDUS-EMMC) - Coordination F-J Richard, Université de Poitiers, France. Doctoral studies of Margot Fortin were funded by the Région NouvelleAquitaine

Both of the funding body had no role in the design of the study and collection, analysis, and interpretation of data and in writing the manuscript.

Availability of data and materials

All raw data are available in the Additional file 2: S2 raw data.

\section{Authors' contributions}

MF, CSG and FJR substantially contributed to the design of the work. MF and $T L$ acquired the data. JM did the statistical analyses. MF and FJR drafted the manuscript. All the authors read and approved the final manuscript.

Ethics approval and consent to participate

Within the context of Directive 2010/63/EU on the protection of animals used for scientific purposes, the European commission decided that most invertebrates, including A. vulgare (Crustacean Isopoda), are excluded from ethical statements. However, we took numerous precautions during our study. Animals used did not come from the field but were reared in our lab. All individuals used for the study were reared in groups and were provided with food ad libitum. The behavioral experiments were not stressful or invasive. After the behavioral experiments, the animals were reared in large boxes in groups.

\section{Consent for publication}

Not applicable.

\section{Competing interests}

The authors declare that they have no competing interests.

\section{Publisher's Note}

Springer Nature remains neutral with regard to jurisdictional claims in published maps and institutional affiliations.

\section{Author details}

${ }^{1}$ Laboratoire Ecologie et Biologie des Interactions, Equipe "Ecologie, Evolution, Symbiose", UMR CNRS 7267, Université de Poitiers, Bat B8-B35, 6 rue Michel Brunet, TSA 51106, F-86073 Poitiers, Cedex 9, France. ${ }^{2}$ Institut de Recherche sur la Biologie de l'Insecte (IRBI), UMR 7261, CNRS, Université de Tours, Tours, France.

Received: 23 July 2018 Accepted: 14 February 2019

Published online: 28 February 2019

References

1. Chapman T, Arnqvist G, Bangham J, Rowe L. Sexual conflict. Trends Ecol Evol. 2003;18(1):41-7.

2. Bateman A. Lntra-sexual selection in Drosophila. Heredity. 1948;2(3):349-68.

3. Arnqvist $\mathrm{G}$, Nilsson $\mathrm{T}$. The evolution of polyandry: multiple mating and female fitness in insects. Anim Behav. 2000;60(2):145-64.

4. Crudgington HS, Siva-Jothy MT. Genital damage, kicking and early death. Nature. 2000;407(6806):855-6.

5. Chapman T, Takahisa M, Smith HK, Partridge L. Interactions of mating, egg production and death rates in females of the Mediterranean fruitfly, Ceratitis capitata. Proc Roy Soc B. 1998;265(1408):1879-94.

6. Makowicz AM, Schlupp I. The direct costs of living in a sexually harassing environment. Anim Behav. 2013;85(3):569-77.

7. Krupa JJ, Sih A. Experimental studies on water strider mating dynamics - spatial variation in density and sex-ratio. Behav Ecol Sociobiol. 1993; 33(2):107-20

8. Chilvers BL, Robertson BC, Wilkinson IS, Duignan PJ, Gemmell NJ. Male harassment of female New Zealand Sea lions, Phocarctos hookeri: mortality, injury, and harassment avoidance. Can J Zool. 2005;83(5):642-8.

9. Miller WJ, Schneider D. Endosymbiotic microbes as adaptive manipulators or arthropod behavior and natural driving sources of host speciation. In: 
Hughes DP, Brodeur J, Thomas F, editors. Host manipulation by parasites. Oxford University Press; 2012. p. 119-37.

10. Cordaux R, Pichon S, Hatira HBA, Doublet V, Grève P, Marcadé I, BraquartVarnier C, Souty-Grosset C, Charfi-Cheikhrouha F, Bouchon D. Widespread Wolbachia infection in terrestrial isopods and other crustaceans. Zookeys. 2012;176:123-31.

11. Werren JH, Windsor D, Guo LR. Distribution of Wolbachia among neotropical arthropods. Proc Roy Soc B. 1995;262(1364):197-204.

12. Sironi M, Bandi C, Sacchi L, DiSacco B, Damiani G, Genchi C. Molecular evidence for a close relative of the arthropod endosymbiont Wolbachia in a filarial worm. Mol Biochem Parasitol. 1995;74(2):223-7.

13. Baldo L, Prendini L, Corthals A, Werren JH. Wolbachia are present in southern African scorpions and cluster with supergroup F. Curr Microbiol. 2007:55(5):367-73.

14. Bouchon D, Rigaud T, Juchault P. Evidence for widespread Wolbachia infection in isopod crustaceans: molecular identification and host feminization. Proc Roy Soc B. 1998;265(1401):1081-90.

15. Werren JH, Baldo L, Clark ME. Wolbachia: master manipulators of invertebrate biology. Nature Rev Microbiol. 2008;6(10):741-51.

16. Braquart-Varnier $C$, Lachat $M$, Herbiniere J, Johnson M, Caubet $Y$, Bouchon D, Sicard M. Wolbachia mediate variation of host immunocompetence. PLoS One. 2008;3(9).

17. Moreau J, Bertin A, Caubet Y, Rigaud T. Sexual selection in an isopod with Wolbachia- induced sex reversal: males prefer real females. J Evol Biol. 2001; 14:388-94.

18. Richard F-J. Symbiotic bacteria influence the odor and mating preference of their hosts. Front Ecol Evol. 2017;5:143.

19. Martin G, Juchault $P$, Legrand J. Mise en évidence d'un micro-organisme intracytoplasmique symbiote de l'oniscoïde Armadillidium vulgare Latr. dont la présence accompagne l'intersexualité ou la féminisation totale des mâles génétiques de la lignée thélygène. CRAS. 1973;276:2313-6.

20. Juchault P, Rigaud T, Mocquard JP. Evolution of sex-determining mechanisms in a wild population of Armadillidium vulgare Latr.(Crustacea, Isopoda): competition between two feminizing parasitic sex factors. Heredity. 1992;69:382-90.

21. Howard H. Length of life of sperms in the woodlouse Armadillidium vulgare Latr. Nature. 1943;152:331.

22. Johnson C. Genetics of red body polymorphism in the isopod, Venezillo evergladensis. J Heredity. 1976;67(3):157.

23. Johnson C. Multiple insemination and sperm storage in the isopod, Venezillo evergladensis. Crustaceana. 1982;42(1):225-32.

24. Moreau J, Seguin S, Caubet Y, Rigaud T. Female remating and sperm competition patterns in a terrestrial crustacean. Anim Behav. 2002;64:569-77.

25. Fortin M, Debenest C, Souty-Grosset C, Richard F-J. Males prefer virgin females, even if parasitized, in the terrestrial isopod Armadillidium vulgare. Ecol Evol. 2018;8:3341-53.

26. Moreau J, Rigaud T. Variable male potential rate of reproduction: high male mating capacity as an adaptation to parasite-induced excess of females? Proc Roy Soc B. 2003;270(1523):1535-40.

27. Rigaud T, Moreau M. A cost of Wolbachia-induced sex reversal and femalebiased sex ratios: decrease in female fertility after sperm depletion in a terrestrial isopod. Proc Roy Soc B. 2004;271(1551):1941-6.

28. Ojanguren AF, Magurran AE. Male harassment reduces short-term female fitness in guppies. Behaviour. 2007;144:503-14.

29. Teseo S, Veerus L, Moreno C, Mery F. Sexual harassment induces a temporary fitness cost but does not constrain the acquisition of environmental information in fruit flies. Biol Lett. 2016;12(1).

30. Magurran AE, Seghers BH. A cost of sexual harassment in the guppy, Poecilia-reticulata. Proc Roy Soc B. 1994;258(1351):89-92.

31. Plath M, Makowicz AM, Schlupp I, Tobler M. Sexual harassment in livebearing fishes (Poeciliidae): comparing courting and noncourting species. Behav Ecol. 2007;18(4):680-8.

32. Joshi D, McFadden MJ, Bevins D, Zhang FR, Xi ZY. Wolbachia strain wAlbB confers both fitness costs and benefit on Anopheles stephensi. Parasites Vect. 2014;7.

33. Fry AJ, Rand DM. Wolbachia interactions that determine Drosophila melanogaster survival. Evolution. 2002;56(10):1976-81.

34. Kohlmeier P, Holländer K, Meunier J. Survival after pathogen exposure in group-living insects: don't forget the stress of social isolation! J Evol Biol. 2016;29(9):1867-72.

35. Waller A, Verdi A. Reproductive patterns of terrestrial isopods (Crustacea, Isopoda, Oniscidea) from Uruguay. Int J Biol. 2016;8(4):12.
36. Cordaux R, Michel-Salzat A, Frelon-Raimond M, Rigaud T, Bouchon D. Evidence for a new feminizing Wolbachia strain in the isopod Armadillidium vulgare: evolutionary implications. Heredity. 2004;93:78-84.

37. Cordaux R, Bouchon D, Grève P. The impact of endosymbionts on the evolution of host sex-determination mechanisms. Trends Genet. 2011;27(8):332-41.
Ready to submit your research? Choose BMC and benefit from:

- fast, convenient online submission

- thorough peer review by experienced researchers in your field

- rapid publication on acceptance

- support for research data, including large and complex data types

- gold Open Access which fosters wider collaboration and increased citations

- maximum visibility for your research: over $100 \mathrm{M}$ website views per year

At $\mathrm{BMC}$, research is always in progress.

Learn more biomedcentral.com/submissions 